\title{
Soumission chimique : Prise en charge toxicologique
}

\section{Toxicological investigations in case of drug-facilitated crimes}

Consensus de la Société Française de Toxicologie Analytique (SFTA)

Établi le 7 novembre 2003

Rédacteur : Pascal KINTZ, IML Strasbourg

Consultable sur le site Internet de la SFTA, http://www.sfta.org

La soumission chimique peut être définie comme l'administration de substances psycho-actives à une personne à des fins délictueuses ou criminelles.

La soumission chimique est un acte criminel dont la prévalence semble être en augmentation, comme le rapporte l'étude de la presse spécialisée. Les statistiques officielles sont bien inférieures au nombre important d'expertises judiciaires pratiquées.

Selon les agresseurs, le produit idéal est celui qui est actif à faible dose (donc aisément incorporable dans une boisson), rapidement soluble en milieu aqueux, sans goût, et dont les effets sont rapides à s'installer, tout en provoquant une amnésie des. faits (amnésie antérograde).

L'administration se fait dans la plupart des cas par voie orale dans les boissons. Le café (probablement du fait de sa couleur et de son amertume naturelle), les jus de fruits et la bière sont a priori les plus fréquemment rencontrés.

Dans ces conditions, il a semblé important que la SFTA étudie ce sujet, afin de proposer des recommandations pour les prélèvements et les analyses toxicologiques.

\section{Quels produits?}

Les produits les plus utilisés sont les suivants :

- l'alcool éthylique

- les benzodiazépines, qu'elles soient à demi-vie courte ou longue et notamment le triazolam, le flunitrazé- pam, le lorazépam, mais aussi le clonazépam, l'alprazolam, le bromazépam et enfin le chlorazépate dipotassique

- des hypnotiques, comme le zolpidem ou la zopiclone - des anesthésiques, comme le GHB ou l'hydrate de chloral, exceptionnellement la kétamine ou le fentanyl

- les dérivés de l'ecstasy (MDMA), le cannabis

- des hallucinogènes tels le LSD, la scopolamine et l'atropine (à partir du Datura), ou les dérivés de la dimethyltryptamine (Ayahuasca)

- des médicaments détournés de leur AMM, comme certains neuroleptiques (chlorpromazine ou lévomépromazine) ou certains anti-histaminiques $\mathrm{H} 1$ à visée sédative (alimémazine, doxylamine, niaprazine), le trihexyphénidyle ou encore le méprobamate

\section{Quels prélèvements ?}

Trois prélèvements doivent être réalisés de façon systématique :

- du sang, prélevé exclusivement sur EDTA (pour éviter la formation in vitro de GHB)

- 3 tubes de $10 \mathrm{ml} \mathrm{(2} \mathrm{pour} \mathrm{l'analyse,} 1$ pour la contre-expertise éventuelle)

- des urines, sur tube sec (type ECBU)

- 2 flacons de $30 \mathrm{ml}$ (1 pour l'analyse, 1 pour la contre-expertise éventuelle, conservés à l'abri de la lumière pour éviter la dégradation du LSD)

- des cheveux, orientés racine-pointe et coupés au ras 
du cuir chevelu en occipital, d'un diamètre équivalent à celui d'un crayon à papier. Il est impératif de prélever les cheveux 3 à 5 semaines après les faits, afin de suivre, par segmentation, les variations de concentrations des produits

- 4 mèches ( 3 pour l'analyse, 1 pour la contreexpertise éventuelle)

L'analyse du sang et des urines doit être effectuée sans délai. En cas contraire, il convient de congeler les prélèvements.

\section{Conservation des prélèvements et durée}

Le sang et les urines sont à conserver au froid $\left(-20^{\circ} \mathrm{C}\right)$.

Les cheveux sont à maintenir au sec, à température ambiante.

Lorsque la justice a été ou s'est saisie de l'affaire, les échantillons devront être conservés jusqu'à l'extinction de toute procédure judiciaire ou recours possible et ne pourront être détruits qu'avec l'accord de l'autorité requérante.

Dans le cas contraire, il est conseillé de conserver tous les échantillons pendant 12 mois.

Une fiche de liaison entre le clinicien réalisant la prise en charge médico-légale et le toxicologue est nécessaire. En complément de l'anamnèse, cette fiche doit comporter l'heure supposée des faits, le moment des prélèvements, le(s) traitement(s) habituel(s) de la victime (en particulier si elle utilise des hypnotiques le soir), une consommation de produits stupéfiants et un éventuel traitement (sédatif, anxiolytique...) depuis les faits. En complément des informations obtenues par l'analyse du sang ou des urines, les investigations toxicologiques à partir des cheveux sont indispensables pour :

- différencier une exposition unique d'un usage chronique. Cette analyse se fait par segmentation de la mèche de cheveux et permet de démontrer si le produit mis en évidence par les analyses sanguines et/ou urinaires correspond à une consommation habituelle ou à une exposition unique. L'avocat du mis en examen pouvant arguer que la victime était utilisatrice du produit retrouvé au moment des faits, voire antérieurement aux faits, seule la discrimination par l'analyse des cheveux permet d'établir la vérité.

- mettre en évidence un xénobiotique lorsque les prélèvements sanguins ou urinaires sont trop tardifs. La soumission chimique implique le plus souvent l'administration d'une seule dose de substances actives à demivie courte. Dans la plupart des cas, lorsque les prélèvements sont tardifs ( $>24$ heures pour le sang ou $>48$ heures pour les urines), les méthodes conventionnelles comme la chromatographie en phase gazeuse couplée à la spectrométrie de masse ou la chromatographie liquide couplée à un détecteur à barrette de diodes ne permettront pas de détecter ces produits. En augmentant la fenêtre de détection à plusieurs semaines, voire plusieurs mois, les cheveux, lorsqu'ils sont analysés par des méthodes de chromatographie couplées à la spectrométrie de masse en tandem (MS/MS), peuvent permettre de mettre en évidence une exposition unique.

\section{Quelles analyses?}

L'analyse toxicologique doit être réalisée le plus rapidement possible. Les benzodiazépines sont des produits chimiquement instables, dont la dégradation peut être totale en quelques jours, en particulier le flunitrazépam ou le clonazepam. Il en est de même pour le LSD. De plus, la formation in vitro du GHB, maintenant bien connue mais mal maîtrisée, est une source de faux positifs et donc d'erreurs judiciaires.

Les dépistages par immunochimie sont à proscrire. De nombreuses substances ne sont pas reconnues par les réactifs disponibles, comme le zolpidem, la zopiclone, le GHB, la scopolamine, la kétamine, les neuroleptiques. Une dose équivalente à une unité galénique de lorazépam, bromazépam, flunitrazépam, clonazépam, zopiclone ou zolpidem n'est jamais détectée par immunochimie dans les urines, quel que soit le moment du prélèvement.

Dans ces conditions, la règle absolue en matière d'analyse toxicologique pour la caractérisation d'une soumission chimique est la mise en place de techniques séparatives et de détections sensibles et spécifiques.

Pour cela, les techniques suivantes doivent être mise en oeuvre :

\section{1 - Analyse du sang et des urines}

- chromatographie en phase gazeuse et détection par ionisation de flamme (GC/FID), pour l'éthanol

- chromatographie en phase gazeuse couplée à la spectrométrie de masse avec introduction par espace de tête (HS/GC/MS), pour les substances volatiles comme l'hydrate de chloral

- chromatographie en phase gazeuse couplée à la spectrométrie de masse (GC/MS) et chromatographie liquide couplée à un détecteur à barrette de diodes (LC/DAD) pour les stupéfiants comme le cannabis, la kétamine, les dérivés de l'ecstasy, le GHB et les médicaments de type neuroleptiques ou anti-histaminiques

- chromatographie en phase liquide couplée à la spectrométrie de masse (LC/MS) pour les hypnotiques comme le zolpidem, la zopiclone et certaines benzodiazépines ou le LSD

- chromatographie en phase liquide ou gazeuse couplée à la spectrométrie de masse en tandem (MS/MS) pour les cas où les prélèvements ont été réalisés tardivement (délai > 36 heures pour le zolpidem, > 84-96 heures pour le flunitrazépam ou le lorazépam) 
Les investigations analytiques recommandées pour le sang et/ou les urines sont les suivantes (par ordre de priorité) :

\author{
Ethanol \\ Cannabis \\ Benzodiazépines et hypnotiques \\ Amphétamines et dérivés \\ GHB \\ Screening large de médicaments \\ Autres stupéfiants \\ Atropine, scopolamine \\ Substances volatiles
}

\section{2 - Analyse des cheveux}

- chromatographie en phase gazeuse couplée à la spectrométrie de masse (GC/MS) pour les stupéfiants

- chromatographie en phase liquide couplée à la spectrométrie de masse en tandem (MS/MS) pour les hypnotiques et les benzodiazépines

- chromatographie en phase gazeuse couplée à la spectrométrie de masse en tandem (MS/MS) pour le GHB et le cannabis en prise unique

- chromatographie en phase gazeuse couplée à la spectrométrie de masse (GC/MS) et chromatographie en phase liquide couplée à un détecteur à barrette de diodes (LC/DAD) pour la recherche large des médicaments

Le protocole analytique recommandé pour les cheveux est le suivant (avec ordre de priorité) :

$\rightarrow$ Sectionner les cheveux en 3 segments (sauf pour le GHB)

- entre 0 (racine) et $2 \mathrm{~cm}$, devrait contenir le xénobiotique si le prélèvement est fait 3 à 5 semaines après les faits

- entre 2 et $4 \mathrm{~cm}$, zone tampon

- et entre 4 et $6 \mathrm{~cm}$, ne devrait pas contenir de xénobiotique

Sont à rechercher sur

- la mèche 1 : benzodiazépines, hypnotiques (LCMS/MS)

- la mèche 2 : stupéfiants + screening médicaments (GC/MS, LC/DAD)

- la mèche 3 : GHB par section de $3 \mathrm{~mm}$ (GC-MS/MS)

Au total, l'équipement de base pour documenter un fait de soumission chimique est le suivant :

- analyse du sang et des urines : GC/FID, LC/DAD, GC/MS, LC/MS

- analyse des cheveux : LC/DAD, GC/MS, LCMS/MS, GC-MS/MS

En cas de plainte déposée, si l'expert judiciaire désigné par les autorités requérantes ne dispose pas de la totalité des techniques analytiques précitées, une co-désignation (adjonction) pour une investigation ponctuelle avec un deuxième expert judiciaire disposant de ces techniques analytiques devra être demandée aux autorités requérantes.

\section{Conclusion}

Chaque cas de soumission chimique (relevant des Assises) doit être documenté au mieux, mais le diagnostic toxicologique est difficile. Souvent, les produits utilisés ont des demi-vies courtes, les concentrations circulantes sont faibles et les prélèvements sont tardifs.

Un système d'assurance qualité, avec des échantillons réels doit être envisagé pour démontrer la capacité des experts spécialisés à réaliser correctement de telles investigations toxicologiques.

\section{Références}

Kintz P., Villain M., Cirimele V., Goullé J.-P., Ludes B. Usage criminel de substances psycho-actives : le problème de la durée de détection. Acta Clin Belgica. $2002 ; 57$, suppl $1: 24-30$.

Wells D. Drug administration and sexual assault : sex in a glass. Sciences \& Justice. 2001 ; 41 : 197-199.

Burnat P., Garcia C., Marc B., Allio I., Perrin M., Ceppa F. Agressions sexuelles et soumissions chimiques, un problème d'actualité. Presse Med. 2002 ; 31 : 705-712.

Djezzar S., Questel F., Dally S. La soumission médicamenteuse. Courrier des Addictions. $2001 ; 3: 164$.

Bismuth C., Dally C., Borron S. Chemical submission : GHB, benzodiazepines and other knock out drops. J Toxicol Clin Toxicol. 1997 ; 35 : 595-598.

Questel F., Bécour B., Dupeyron J.P., Galliot-Guillet M., Diamant-Berger $O$. Soumission médicamenteuse : 4 ans d'expérience aux UMJ de Paris. J Med Leg Droit Med. 2000 ; 43 : $459-465$.

Ghysel M.-H., Pépin G., Kintz P. La soumission médicamenteuse. Toxicorama. $1998 ; 10: 126-128$.

ElSohly M., Lee L., Holzhauer L., Salamone S. Analysis of urine samples in cases of alleged sexual assault. In : Salamone S., ed. Benzodiazepines and GHB. Detection and Pharmacology. Totowa : Humana Press. 2001 : 127-144.

LeBeau M., Andollo W., Hearn L. et al. Recommendations for toxicological investigations of drug-facilitated sexual assaults. J Forensic Sci. $1999 ; 44$ : 227-230.

Kintz P., Cirimele V., Jamey C., Ludes B. Testing for GHB in hair by GC/MS/MS after a single exposure. Application to document sexual assaults. J Forensic Sci. $2003 ; 48$ : 195-200.

Pépin G., Gaillard Y. A propos de deux cas d'utilisation originale de la soumission chimique. Ann Toxicol Anal. $2001 ; 13$ : $122-123$.

Pépin G., Gaillard Y., Chèze M., Goullé JP. Le GHB dans les phanères : résultats et interprétation. J Med Leg Droit Med. $2003 ; 46: 93-100$.

Drug Facilitated Sexual Assault, eds. LeBeau M. et Mozayani A. San Diego : Academic Press. 2001, pp 1-326.

La soumission chimique : numéro spécial des Annales de Toxicologie Analytique, $2002 ; 14$ : numéro 4, pp 359-425. 


\section{Soumission chimique : étude expérimentale in vivo sur volontaires sains}

- lorazépam : 1 comprimé à $2,5 \mathrm{mg}$ de TEMESTA®

- bromazépam : 1 comprimé baguette quadrisécable à $6 \mathrm{mg}$ de LEXOMIL®

- flunitrazépam : 1 comprimé à $1 \mathrm{mg}$ de ROHYPNOL®

- zolpidem : 1 comprimé à $10 \mathrm{mg}$ de STILNOX®

- clonazépam : 1 comprimé à 2 mg de RIVOTRIL®

- zopiclone : 1 comprimé à 7,5 mg d'IMOVANE®

Durée de détectabilité dans les urines

\begin{tabular}{|c|c|c|c|c|c|c|}
\hline Molécules & $\begin{array}{l}\text { Immuno sauf } \\
\text { CEDIA }\end{array}$ & CEDIA & LC-DAD & GC-MS & LC-MS & LC-MS/MS \\
\hline $\begin{array}{l}\text { Lorazépam } \\
\text { (Temesta) }\end{array}$ & Non détecté & $96 \mathrm{~h}$ & Non détecté & $84 \mathrm{~h}$ & $84 h$ & $144 h^{* *}$ \\
\hline OH-bromazépam & Non détecté & 1 & $36 \mathrm{~h}$ & $24 \mathrm{~h}$ & $108 \mathrm{~h}$ & $144 \mathrm{~h}$ \\
\hline $\begin{array}{l}\text { Bromazépam } \\
\left.\text { (Lexomil }^{\circledR}\right)\end{array}$ & Non détecté & $48 \mathrm{~h}$ & $12 \mathrm{~h}$ & $72 \mathrm{~h}$ & $84 h$ & $144 \mathrm{~h}^{* * *}$ \\
\hline $\begin{array}{l}\text { Zopiclone } \\
\left.\text { (Imovane }^{\circledR}\right)\end{array}$ & Non détecté & Non detecté & $12 \mathrm{~h}$ & dégradée & $36-48 \mathrm{~h}$ & $144 \mathrm{~h} * *$ \\
\hline $\begin{array}{c}\text { 7-NH2 } \text {-flunitrazépam } \\
\left.\text { (Rohypnol }^{\circledR}\right)\end{array}$ & Non détecté & $48 \mathrm{~h}$ & $36 \mathrm{~h}$ & $48 \mathrm{~h}$ & $96 \mathrm{~h}$ & $144 h^{* * *}$ \\
\hline $\begin{array}{l}\text { Zolpidem } \\
\left(\text { Stilnox }^{\circledR}\right)\end{array}$ & Non détecté & Non détecté & $24 \mathrm{~h}$ & $12 \mathrm{~h}$ & $36-60 h^{*}$ & $96-144 \mathrm{~h}$ \\
\hline $\begin{array}{c}\text { 7- } \mathrm{NH}_{2} \text {-clonazépam } \\
\text { (Rivotril }^{\mathbb{}} \text { ) }\end{array}$ & Non détecté & $96 \mathrm{~h}$ & $12 \mathrm{~h}$ & $132 \mathrm{~h}$ & $144 \mathrm{~h}$ & $144 \mathrm{~h}^{* *}$ \\
\hline
\end{tabular}

* : zolpidem métabolite acide

** : délai minimum

Concentrations mesurées dans les cheveux recueillis 3 à 5 semaines après l'exposition (Segment proximal)

\footnotetext{
- lorazépam : $<1 \mathrm{pg} / \mathrm{mg}$

- bromazépam : $1-5 \mathrm{pg} / \mathrm{mg}$

- flunitrazépam : $0,5-5 \mathrm{pg} / \mathrm{mg}$ (7- $\mathrm{NH}_{2}$-flunitrazépam)

- zolpidem : $1-10 \mathrm{pg} / \mathrm{mg}$

- clonazépam : $1-10 \mathrm{pg} / \mathrm{mg}$ (7- $\mathrm{NH}_{2}$-clonazépam)

- zopiclone : $5-20 \mathrm{pg} / \mathrm{mg}$
} 